\title{
Chemoradiotherapy Using Carboplatin plus Paclitaxel versus Cisplatin plus Fluorouracil for Esophageal or Gastroesophageal Junction Cancer
}

\author{
Di Maria Jiang ${ }^{a}$ Hao-Wen Sim ${ }^{a}$ Osvaldo Espin-Garciab Bryan A. Chan ${ }^{a}$ \\ Akina Natori ${ }^{a} \quad$ Charles H. Lim ${ }^{a}$ Stephanie Moignard ${ }^{a}$ Eric X. Chen ${ }^{a}$ \\ Geoffrey Liu ${ }^{a} \quad$ Gail Darling $^{c}$ Carol J. Swallow ${ }^{d}$ Savtaj Brard James Brierley ${ }^{\mathrm{e}}$ \\ Jolie Ringash ${ }^{\mathrm{e}}$ Rebecca Wong ${ }^{\mathrm{e}}$ John Kim $^{\mathrm{e}}$ Patrik Rogalla $^{\mathrm{f}}$ \\ Sara Hafezi-Bakhtiari ${ }^{9}$ Jennifer J. Knox ${ }^{a}$ Raymond W. Jang ${ }^{a} \quad E^{\prime}$ Elena Elimova ${ }^{a}$ \\ aDepartment of Medical Oncology, Princess Margaret Cancer Centre, University Health Network, University of \\ Toronto, Toronto, ON, Canada; ${ }^{b}$ Department of Biostatistics, Princess Margaret Cancer Centre, University Health \\ Network, Dalla Lana School of Public Health, University of Toronto, Toronto, ON, Canada; 'Division of Thoracic \\ Surgery, Department of Surgery, Toronto General Hospital, University Health Network, University of Toronto, \\ Toronto, ON, Canada; d Department of Surgical Oncology, Mount Sinai Hospital/Princess Margaret Cancer Centre,

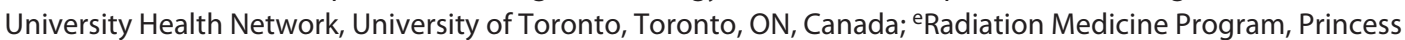 \\ Margaret Cancer Centre, Ontario Cancer Institute, University Health Network, University of Toronto, Toronto, \\ ON, Canada; ${ }^{\mathrm{f} J o i n t ~ D e p a r t m e n t ~ o f ~ M e d i c a l ~ I m a g i n g, ~ U n i v e r s i t y ~ o f ~ T o r o n t o, ~ T o r o n t o, ~ O N, ~ C a n a d a ; ~} 9$ Department of \\ Pathology, Toronto General Hospital, University Health Network, University of Toronto, Toronto, ON, Canada
}

\section{Keywords}

Esophageal neoplasms · Chemoradiotherapy · Cisplatin · Carboplatin · Survival analysis

\begin{abstract}
Background: Trimodality therapy (TMT) with neoadjuvant chemoradiotherapy (nCRT) using concurrent carboplatin plus paclitaxel (CP) followed by surgery is the standard of care for locoregional esophageal or gastroesophageal junction (GEJ) cancers. Alternatively, nCRT with cisplatin plus fluorouracil (CF) can be used. Definitive chemoradiotherapy (dCRT) with CP or CF can be used if surgery is not planned. In the absence of comparative trials, we aimed to evaluate outcomes of CP and CF in the settings of TMT and dCRT. Methods: A single-site, retrospective cohort study was conducted
\end{abstract}

karger@karger.com www.karger.com/ocl

Karger $\stackrel{\text { ' }}{5}$

GOPEN ACCESS
(C) 2020 The Author(s)

Published by S. Karger AG, Basel

This is an Open Access article licensed under the Creative Commons Attribution-NonCommercial-4.0 International License (CC BY-NC) (http://www.karger.com/Services/OpenAccessLicense), applicable to the online version of the article only. Usage and distribution for commercial purposes requires written permission. at the Princess Margaret Cancer Centre to identify all patients who received CRT for locoregional esophageal or GEJ cancer. Overall survival (OS) and disease-free survival (DFS) were assessed using the Kaplan-Meier method and multivariable Cox regression model. The inverse probability treatment weighting (IPTW) method was used for sensitivity analysis. Results: Between 2011 and 2015, 93 patients with esophageal (49\%) and GEJ (51\%) cancers underwent nCRT ( $n=67 ; 72 \%)$ or dCRT $(n=26 ; 28 \%)$. Median age was 62.3 years and $74 \%$ were male. Median follow-up was 23.9 months. Comparing $\mathrm{CP}$ to $\mathrm{CF}$ in the setting of TMT, the OS and DFS rates were similar. In the setting of dCRT, CP was associated with significantly inferior 3 -year OS (36 vs. 63\%;

D.M. Jiang and H.-W. Sim are co-first authors. 
$p=0.001 ; \mathrm{HR} 3.1 ; 95 \% \mathrm{Cl}: 1.2-7.7)$ and DFS ( 0 vs. $41 \% ; p=$ 0.004; HR 3.6; $95 \%$ Cl: $1.4-8.9$ ) on multivariable and IPTW sensitivity analyses. Conclusions: TMT with CF and CP produced comparable outcomes. However, for dCRT, CF may be a superior regimen.

(c) 2020 The Author(s)

Published by S. Karger AG, Basel

\section{Introduction}

Locally advanced esophageal and gastroesophageal junction (GEJ) cancers are potentially highly lethal malignancies. Surgical resection alone has suboptimal cure rates of only $<40 \%$ and is associated with significant risks of both local and distant relapse [1]. This is at least partially related to the locally advanced stage on presentation for the majority of patients, as considerable tumor penetration into the esophageal lumen or invasion into adjacent structures is required to induce symptoms of dysphagia or pain [2].

In this clinical context, various perioperative treatment strategies have been evaluated to improve survival outcomes. Historically, trimodality therapy (TMT) consisting of neoadjuvant chemoradiotherapy (nCRT) of 50 Gy with concurrent cisplatin plus infusional fluorouracil (CF) followed by resection was a standard approach, as shown in the CALGB 9781 trial [3, 4]. In 2012, the CROSS trial evaluating nCRT of 41.4 Gy with concurrent carboplatin plus paclitaxel (CP) followed by surgery established the current standard of care for resectable patients [1]. The pathologic complete response (pCR) rate was $29 \%$, and the 5-year overall survival (OS) rate was $47 \%$, versus $33 \%$ with surgery alone [5]. CROSS reported a more favorable toxicity profile of $\mathrm{CP}$, with fewer severe adverse events than with CF. Definitive chemoradiotherapy (dCRT; deferring surgery for salvage therapy upon local recurrence) using radiotherapy doses of 50.4 Gy with concurrent $\mathrm{CF}$ is another approach supported by multiple phase III trials [6,7], and is used mainly in patients with inoperable or unresectable disease, or in those who refuse surgery [8]. dCRT with CP is also a recommended option as per the US National Comprehensive Cancer Network (NCCN) guideline [8].

In the absence of head-to-head comparisons, the optimal nCRT and dCRT regimens remain undefined. This study aimed to compare the outcomes of the two CRT regimens in patients with locally advanced esophageal or GEJ cancer, i.e., $\mathrm{CP}$ concurrent with $41.4 \mathrm{~Gy}$ versus $\mathrm{CF}$ with $50 \mathrm{~Gy}$ of radiotherapy in the setting of TMT, and CP versus CF with $50 \mathrm{~Gy}$ of radiotherapy in the setting of dCRT.

\section{Subjects and Methods}

\section{Study Cohort and Data Collection}

From the Princess Margaret Cancer Centre institutional registry, we identified consecutive patients with esophageal and GEJ cancer who underwent nCRT or dCRT between 2011 and 2015. Eligible patients were at least 18 years of age, had histological confirmation of esophageal or GEJ cancer without evidence of distant metastases at initial presentation, and received curative-intent therapy. Patients who received radiotherapy doses $<40 \mathrm{~Gy}$ or adjuvant CRT were excluded. Patients with cervical esophageal cancers were excluded, as these patients were typically treated with 70 Gy of radiotherapy concurrent with high-dose cisplatin.

After initial diagnosis, patients had standard clinical staging investigations including esophagogastroduodenoscopy $+/-$ endoscopic ultrasound, computerized tomography (CT) scanning of the chest/abdomen/pelvis, and positron emission tomography. All patients had clinical and pathologic TNM staging according to the American Joint Committee on Cancer (AJCC)/Union for International Cancer Control (UJCC) system 6th edition and 7th edition, respectively.

Prior to data collection from the electronic medical records, all extractors underwent data dictionary training. All extracted data were verified by a second investigator (E.E.) for quality assurance. Discrepancies were discussed with a third investigator to reach a consensus.

\section{Chemoradiotherapy}

Curative treatment options were discussed by a multidisciplinary team consisting of radiologists, surgical oncologists, radiation oncologists, medical oncologists, and pathologists whenever possible. For patients with locoregionally advanced disease, the treatment options consisted of TMT or dCRT. The recommended treatment modality was based on resectability, primary tumor location, histology subtype, anticipated treatment-related morbidity, and patient preference. At our institution, TMT was recommended for patients with resectable esophageal and Siewert class I GEJ cancers and for select Siewert class II tumors based on their proximal extension. Siewert class III tumors were generally treated with perioperative chemotherapy rather than nCRT, similar to gastric cancer. dCRT was recommended for patients with proximal esophageal squamous cell carcinoma (SCC), unresectable or borderline resectable disease (with high risk of a positive resection margin), or an unacceptable risk of surgical morbidity.

At our institution, prior to 2013, the preferred nCRT regimen was $50 \mathrm{~Gy}$ over 25 fractions with concurrent CF (cisplatin $25 \mathrm{mg} /$ $\mathrm{m}^{2}$ and fluorouracil $1,000 \mathrm{mg} / \mathrm{m}^{2}$ on days $1-4$ in weeks 1 and 5). After 2013, the preferred nCRT regimen was 41.4 Gy over 23 fractions with concurrent CP (weekly carboplatin AUC $2 \mathrm{mg} /$ $\mathrm{mL} / \mathrm{min}$ and paclitaxel $50 \mathrm{mg} / \mathrm{m}^{2}$ ) according to the CROSS regimen [1]. Subsequent surgery was performed 6-12 weeks after completion of nCRT if the patient was fit and restaging chest/ abdomen/pelvis CT did not demonstrate unresectable or metastatic disease. Response to nCRT was classified by tumor regression grade, with pCR defined as absence of residual carcinoma on surgical pathology.

For dCRT, radiotherapy of 50 Gy over 25 fractions was delivered to the primary tumor and enlarged lymph nodes by volumetric modulated arc therapy, intensity-modulated radiation therapy, or conformal techniques, with a planning target volume of $3-4 \mathrm{~cm}$
50

Oncology 2021;99:49-56

DOI: $10.1159 / 000510446$
Jiang et al. 
proximally/distally and $1 \mathrm{~cm}$ radially from the gross tumor volume. Dose escalation beyond 50 Gy was not common (4 patients), although considered in patients where surgical salvage was unlikely to be a future option. Either concurrent CF or CP (the latter being increasingly utilized after 2013) was given at the same chemotherapy dosing as outlined above.

\section{Relapse Patterns}

Upon first relapse, complete restaging including chest/abdomen/pelvis CT was performed. Relapse patterns were characterized as locoregional recurrence (LRR) or distant recurrence (DR) or both. LRR occurred near the surgical anastomosis, within the gastroesophageal lumen, or in regional lymph nodes. DR was any recurrence beyond locoregional, and was subcategorized as nonvisceral (bone, peritoneum, or both), visceral (any other site including distant lymph nodes), or both nonvisceral and visceral. Histological confirmation of recurrence was obtained at the discretion of the treating physician.

\section{Statistical Analysis}

Treatment groups were compared using Wilcoxon's rank-sum tests and Fisher's exact tests for numerical and categorical data, respectively. Time-to-event data were analyzed by the KaplanMeier method. OS, disease-free survival (DFS), and time to relapse (TTR) were calculated from the date of initial diagnostic biopsy, and assessed via univariable and multivariable Cox proportionalhazards regression adjusting for age and radiotherapy dose received ( 50 vs. $<50 \mathrm{~Gy}$ ). Due to the limited sample size, additional variables were not adjusted to avoid overfitting [9]. To allow for adjustment of additional potential confounders, we also performed sensitivity analyses using a logistic regression inverse probability treatment weighting (IPTW) model matching patients who underwent concurrent $\mathrm{CF}$ and $\mathrm{CP}$ in the overall study cohort. The pretreatment variables included in the model were: age, gender, histology, Eastern Cooperative Oncology Group (ECOG) performance status, Charlson comorbidity index (CCI), Siewert class, tumor grade, and surgical intent (nCRT or dCRT).

All statistical analyses were performed using $\mathrm{R}$ version 3.5.2 ( $\mathrm{R}$ Core Team 2014, Vienna, Austria). All $p$ values were two-sided, and $p<0.05$ was considered statistically significant.

\section{Results}

\section{Patient and Treatment Characteristics}

Between 2011 and 2015, 93 consecutive patients who met the study criteria were identified and retrospectively reviewed. Median age was 62.3 years (range 29.6-84.4) and $74 \%$ were male. Patients included were predominantly non-Asian (94\%). Node-positive disease on clinical staging was present in $61 \%$ of the patients. Histology included adenocarcinoma (62\%) and SCC (37\%).

Table 1 shows the baseline characteristics of the overall study cohort. The planned curative treatment strategies included TMT ( $n=67 ; 72 \%)$ and dCRT $(n=26 ; 28 \%)$. The majority of patients who received TMT had adeno- carcinoma on histology (75\%). The nCRT regimens used in TMT included CP $(n=32 ; 48 \%)$ and CF $(n=35 ; 52 \%)$. Among the patients who completed TMT $(n=57)$, those in the $\mathrm{CP}$ group were older than those in the CF group (median age 66.4 vs. 56.3 years; $p<0.001$ ), while ECOG performance status, CCI, Siewert class, histology, and tumor grade were similar. The median number of lymph nodes resected at the time of surgery was 21 (range 4-51). As shown in Table 1, the majority of patients achieved clear margins. Node-positive disease on surgical pathology was present in $44 \%$ of the patients who underwent resection, with similar rates in the $\mathrm{CP}$ and $\mathrm{CF}$ groups.

There were 10 patients (11\%) who received nCRT but did not subsequently undergo resection to complete planned TMT due to either toxicity from nCRT $(n=3)$ or disease progression $(n=7)$ (Appendix 1). Among these 10 patients, 5 received nCRT with CF, 5 received it with $\mathrm{CP}$, and 9 had a adenocarcinoma histology.

Documented reasons for not undergoing surgical resection in the dCRT subcohort included proximal tumor location ( $50 \%$ ); age $\geq 80$ years, comorbidities, or poor performance status (27\%); and extent of nodal disease (24\%) (Appendix 1). The majority of patients who received dCRT had SCC tumors (69\%). All patients completed the planned 50 Gy of radiotherapy, except for 1 patient. The dCRT regimens included $\mathrm{CP}(n=8 ; 31 \%)$ and $\mathrm{CF}(n=18$; 69\%). Patients who received dCRT with CP were older (median age 74.2 vs. 63.1 years) and had a poorer ECOG performance status ( 75 vs. $50 \%$ ECOG performance status $\geq 1$ ), CCI ( 38 vs. $17 \% \mathrm{CCI} \geq 2$ ), more frequent adenocarcinoma histology (50 vs. $22 \%$ ), GEJ primaries (38 vs. $11 \%$ ), and grade 3 tumors (62 vs. $22 \%$ ), although the differences were not statistically significant in this small subcohort. Two patients underwent salvage surgical resection ( 1 in the CP group and 1 in the CF group) for presumed residual disease after dCRT, with surgical pathology showing pT1aN0 and absence of residual disease, respectively.

\section{Treatment Outcomes}

In the TMT subcohort, the pCR rate was $27 \%(8 / 30)$ in the CF group and 15\% (4/27) in the CP group. Both adenocarcinoma and SCC patients achieved PCR with either CF or CP.

The median follow-up in the overall cohort was 23.9 months (28.9 and 15.3 months in the TMT and the dCRT subcohorts, respectively), and 55.5 months among the patients who were alive at the last follow-up. The overall cohort had 59 deaths $(63 \%)$. Among the patients treated with TMT, the 5-year OS and DFS rate was $41 \%$ (95\% CI: 
Table 1. Baseline characteristics of the overall study cohort

\begin{tabular}{|c|c|c|c|}
\hline & $\mathrm{CF}(n=53)$ & $\mathrm{CP}(n=40)$ & $p$ value \\
\hline Age, years & & & 0.0067 \\
\hline Median (range) & $59(29.6,84.4)$ & $65.4(34.3,84.0)$ & \\
\hline Male & $37(70)$ & $32(80)$ & 0.34 \\
\hline ECOG performance status & & & 0.93 \\
\hline 0 & $22(42)$ & $18(45)$ & \\
\hline $1-2$ & $31(58)$ & $22(55)$ & \\
\hline Charlson comorbidity index & & & 0.087 \\
\hline 0 & $38(72)$ & $20(50)$ & \\
\hline 1 & $10(19)$ & $11(28)$ & \\
\hline$\geq 2$ & $5(9)$ & $9(22)$ & \\
\hline Year of diagnosis & & & $<0.001$ \\
\hline$\leq 2012$ & $38(72)$ & $1(2)$ & \\
\hline 2013-2015 & $15(28)$ & $39(98)$ & \\
\hline Surgical resection & & & 0.52 \\
\hline No & $21(40)$ & $13(32)$ & \\
\hline Yes & $32(60)$ & $27(68)$ & \\
\hline Primary tumor location & & & 0.3 \\
\hline Esophagus & $29(55)$ & $17(42)$ & \\
\hline GEJ & $24(45)$ & $23(57)$ & \\
\hline Siewert class I & $14(26)$ & $17(42)$ & \\
\hline Siewert class II & $10(19)$ & $3(8)$ & \\
\hline Siewert class III & $0(0)$ & $3(8)$ & \\
\hline Histology & & & 0.053 \\
\hline Adenocarcinoma & $29(55)$ & $30(75)$ & \\
\hline Squamous & $24(45)$ & $10(25)$ & \\
\hline Tumor grade & & & 0.012 \\
\hline 1, well-differentiated & $7(13)$ & $2(5)$ & \\
\hline 2, moderately differentiated & $17(32)$ & $12(30)$ & \\
\hline 3 , poorly differentiated & $15(28)$ & $23(57)$ & \\
\hline Unavailable & $14(26)$ & $3(8)$ & \\
\hline Length of tumor & & & 0.32 \\
\hline$<5 \mathrm{~cm}$ & $9(23)$ & $13(34)$ & \\
\hline$\geq 5 \mathrm{~cm}$ & $30(77)$ & $25(66)$ & \\
\hline Unavailable & $14(26)$ & $2(5)$ & \\
\hline Radiation dose & & & $<0.001$ \\
\hline$<50$ & $5(11)$ & $33(82)$ & \\
\hline$\geq 50$ & $42(89)$ & $7(18)$ & \\
\hline Unavailable & $6(11)$ & 0 & \\
\hline Clinical TNM staging & & & 0.86 \\
\hline Tx-2N0M0 & $5(10)$ & $4(10)$ & \\
\hline T3-4N0M0 & $13(26)$ & $11(28)$ & \\
\hline Tx-1N1M0 & $6(12)$ & $6(15)$ & \\
\hline T2N1M0 & $6(12)$ & $2(5)$ & \\
\hline T3-4N1M0 & $20(40)$ & $17(42)$ & \\
\hline Unavailable & $3(6)$ & 0 & \\
\hline
\end{tabular}

CF, cisplatin plus 5-fluorouracil; CP, cisplatin plus paclitaxel; ECOG, Eastern Cooperative Oncology Group; GEJ, gastroesophageal junction.
28-56) and 30\% (95\% CI: 20-46), respectively. The OS and DFS rate at 3 years was $57 \%$ (95\% CI: $45-72$ ) and $41 \%$ (95\% CI: 30-56), respectively. Among the patients treated with dCRT, the 3-year OS and DFS rate was 46\% (95\% CI: 29-71) and 28\% (95\% CI: 15-53), respectively. Patients who received nCRT but not subsequent surgery had significantly inferior OS and DFS, with a median OS of only 9.9 months.

Figure 1 compares the survival outcomes for the patients treated with CP and CF in the TMT and dCRT co- 


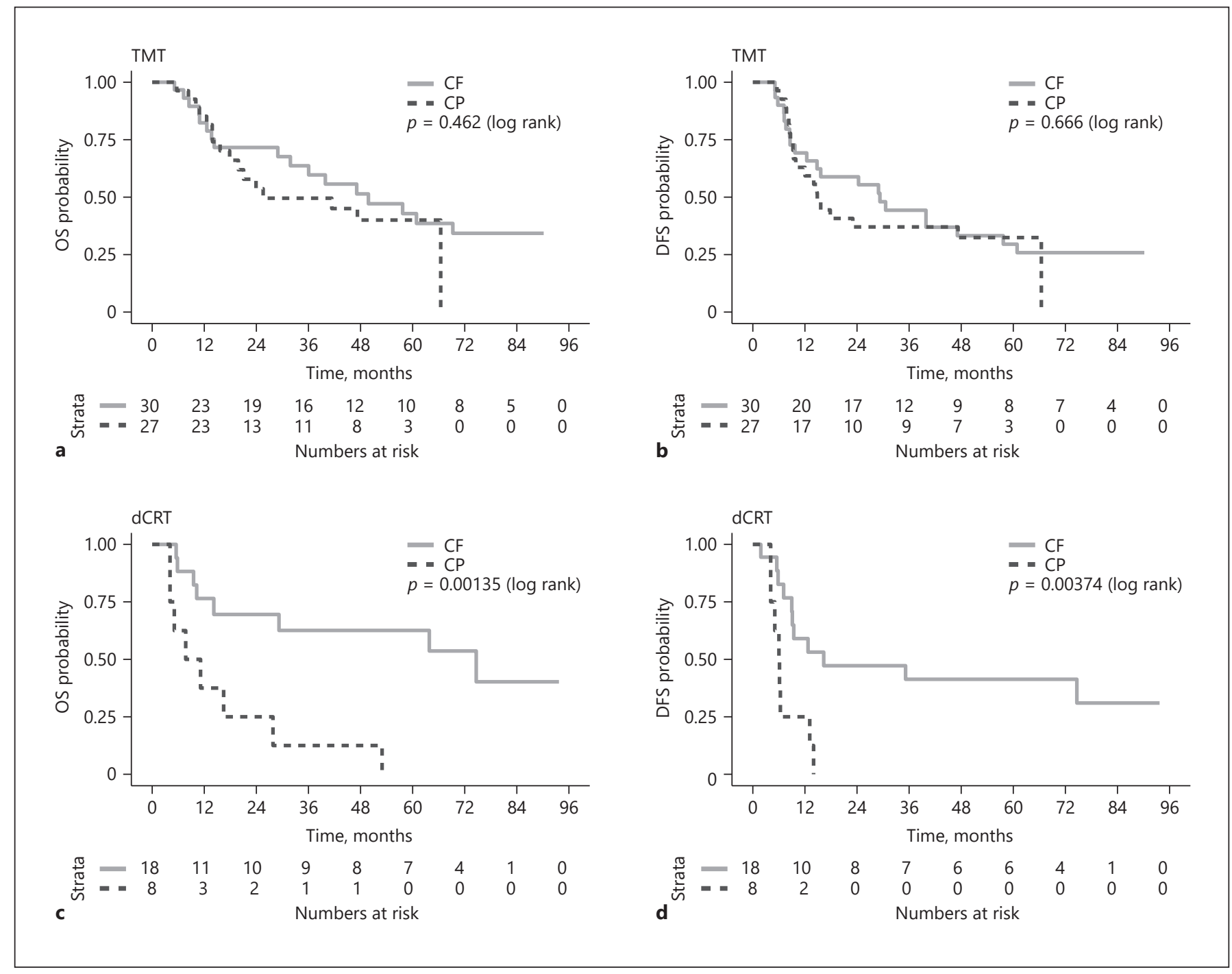

Fig. 1. Kaplan-Meier curves of the OS and DFS of patients who received TMT and dCRT by concurrent chemotherapy regimen. a OS in the TMT cohort. b DFS in the TMT cohort. $\mathbf{c}$ OS in the dCRT cohort. d DFS in the dCRT cohort. OS, overall survival; DFS, disease-free survival; TMT, trimodality therapy; dCRT, definitive chemoradiotherapy; $\mathrm{CF}$, cisplatin plus fluorouracil; $\mathrm{CP}$, carboplatin plus paclitaxel.

horts. In the TMT cohort (Fig. 1a, b), the two nCRT regimens produced similar 3-year OS (64\% with CF vs. $50 \%$ with CP; $p=0.46)$ and DFS rates (44\% with CF vs. $37 \%$ with $\mathrm{CP} ; p=0.67)$. The OS ( 43 vs. $40 \%)$ and DFS rates (30 vs. $32 \%)$ at 5 years were also similar. The sensitivity analysis using IPTW modeling showed similar results comparing CP with CF (HR for OS 1.4, 95\% CI: 0.6-3.2; HR for DFS 1.1, 95\% CI: $0.5-2.3)$. In the 10 patients who had nCRT but no subsequent surgery, nCRT with CP and CF also had similar OS and DFS (data not shown). As shown in Table 2, none of the clinicopathologic variables were associated with survival outcomes in the TMT group on

Chemoradiotherapy for Esophageal Cancer univariable analysis. On multivariable analysis, however, a radiotherapy dose of 50 Gy was associated with improved DFS (HR 0.3; 95\% CI: $0.1-0.9 ; p=0.03$ ) but not OS.

Compared to dCRT with CF, CP produced inferior survival outcomes. As shown in Figure $1 \mathrm{c}$ and d, the 3-year OS rate was 63\% (95\% CI: 43-92) for CF versus $12 \%$ (95\% CI: $2-78)$ for CP $(p=0.001)$. The DFS rate at 3 years was $41 \%$ (95\% CI: $23-73)$ versus $0 \%$ (95\% CI not available) for CF versus $\mathrm{CP}$, respectively $(p=0.004)$. In patients who did not receive surgery, use of $\mathrm{CP}$, adenocarcinoma histology, a GEJ primary, and a radiotherapy 
Table 2. Associations of DFS and OS in the TMT and nonsurgical cohorts

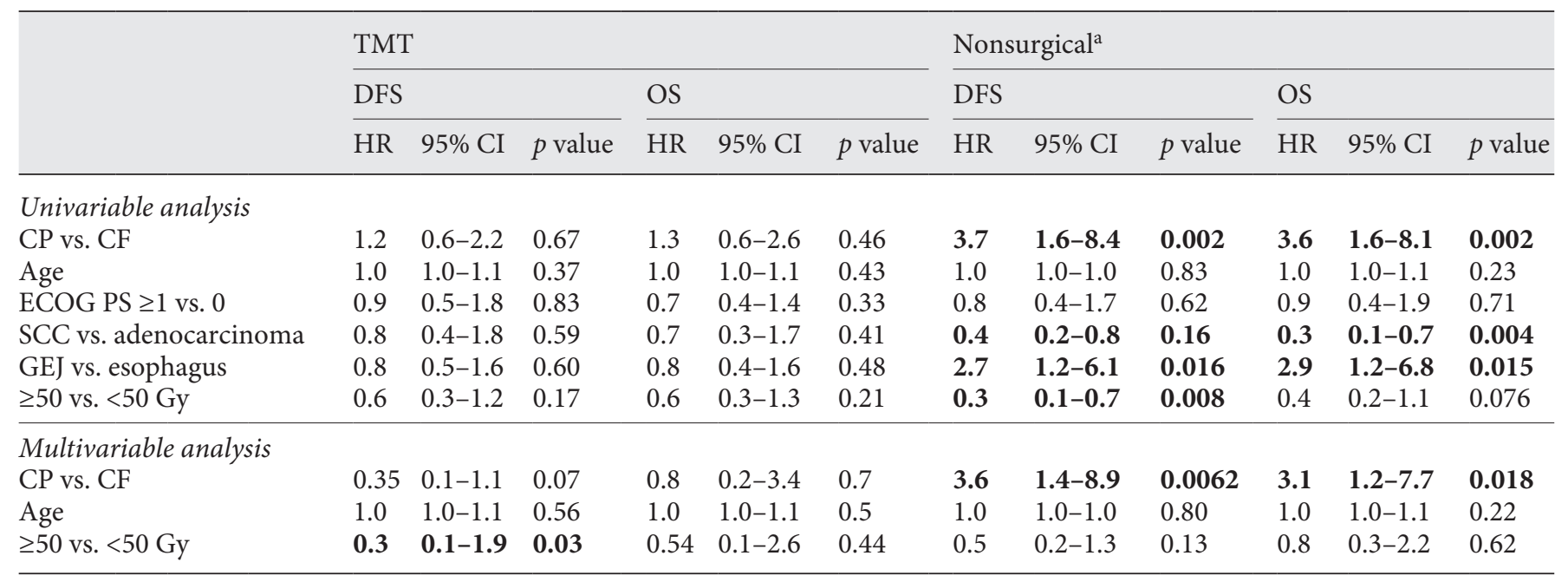

Bold type denotes significance. OS, overall survival; DFS, disease-free survival; TMT, trimodality therapy; CF, cisplatin plus fluorouracil; CP, carboplatin plus paclitaxel; ECOG PS, Eastern Cooperative Oncology Group performance status; SCC, squamous cell carcinoma; GEJ, gastroesophageal junction. ${ }^{\text {a }}$ The nonsurgical cohorts included patients who received definitive chemoradiotherapy and neoadjuvant chemoradiotherapy, without subsequent surgery. These patients were combined to increase statistical power for this analysis.

dose $<50$ Gy were associated with inferior survival outcomes (Table 2). The negative association of CP in this subcohort remained after multivariable analysis adjusting for age and radiotherapy dose delivered, with an HR of 3.1 (95\% CI: 1.2-7.7; $p=0.018$ ) for OS and an HR of 3.6 (95\% CI: $1.4-8.9 ; p=0.0062$ ) for DFS. Sensitivity analysis using IPTW modeling on the nonsurgical cohort supported CP's association with inferior OS (HR 4.3; 95\% CI: $1.8-12.5)$ and DFS (HR 4.3; 95\% CI: 2.1-17.0) compared to $\mathrm{CF}$.

\section{Relapse Patterns}

Among the patients who relapsed in the TMT cohort $(n=29)$, the median time to relapse was 30.6 months (95\% CI: $14.8-57.9)$. The majority had DR (84\%). The relapse patterns were similar in the $\mathrm{CF}$ and $\mathrm{CP}$ groups of the TMT cohort. In the dCRT subcohort, the CP group $(n=3 ; 38 \%)$ seemed to have a higher LRR rate than the CF group ( $n=1 ; 6 \%)$; however, this comparison was based on a small number of patients.

\section{Discussion}

In this study, the two nCRT regimens yielded similar OS and DFS rates and similar survival outcomes to those in the CROSS trial and other contemporary series $[1,10$,
11]. These results, as well as the favorable tolerability of $\mathrm{CP}$, further support $\mathrm{CP}$ as the nCRT regimen of choice. In the setting of dCRT, CP seemed to yield significantly inferior survival, with an $\mathrm{HR}>3$ despite adjusting for age and radiotherapy doses received and additional baseline characteristics using IPTW modeling. The OS data for the dCRT subcohort mirror results from previous reports [12]. The drastically inferior outcomes of the $\mathrm{CP}$ regimen seen highlight the need for caution before adopting $\mathrm{CP}$ as a preferred approach in dCRT.

While dCRT of 50 Gy with CF is established as the standard of care $[6,7], \mathrm{CP}$ has been increasingly utilized without supporting prospective data [12] in accordance with recommendations by the US NCCN guideline [8]. The literature comparing $\mathrm{CP}$ to $\mathrm{CF}$ has been extremely limited for Western patients receiving dCRT, with two comparative retrospective studies, to our knowledge, which published mixed results [12-14]. Both studies found comparable survival outcomes with $\mathrm{CP}$ and $\mathrm{CF}$, but the median OS of the CF groups was significantly shorter (16.1 months [13] and 24.2 months [14] compared to the 74.6 months in our study). Several possibilities exist, the most important being the inclusion of M1 disease as per the AJCC 6th edition (metastasis in celiac lymph nodes for tumors of the lower thoracic esophagus, and cervical nodes for tumors of the upper thoracic esophagus) in one of the prior studies (23\%) [13]. Our 
study did not include patients with celiac nodal metastases at presentation. The second study reported significant differences between the two groups with respect to the median radiotherapy dose delivered: $54 \mathrm{~Gy}$ for $\mathrm{CF}$ and $59.4 \mathrm{~Gy}$ for CP [14]. Both of these doses are considerably higher than the conventional standard of $50 \mathrm{~Gy}$, and may cause a higher degree of toxicity without any additional advantage in survival $[7,15,16]$.

Interestingly, dCRT with CF seemed to benefit both OS and local control compared to CP in our study. It is possible that in the absence of surgery, cisplatin synergizes better with radiotherapy than carboplatin-based therapies, and further decreases local relapse in this disease. There is also high-level evidence for lung as well as head and neck cancers supporting concurrent cisplatin over carboplatin in the setting of dCRT $[17,18]$.

There are several limitations to this study. Because we excluded patients who received radiotherapy doses $<40$ $\mathrm{Gy}$, we were not able to assess whether concurrent $\mathrm{CP}$ and $\mathrm{CF}$ regimens had comparable CRT completion rates. This was not the objective of our study. Our sample size has limited power, and there were considerable imbalances in baseline variables. No definitive conclusions can be drawn due to these limitations. We could not perform extended multivariable analyses given the small sample size adjusting for the nonsignificant differences in histology, performance status, comorbidities, or other unknown confounders. Due to the retrospective nature of the study, selection bias and unknown confounders may have affected the comparative survival outcomes. In our dCRT subcohort, more patients in the CP group had an adenocarcinoma histology (50 vs. $22 \%$ ) and $<50$ Gy of radiotherapy (38 vs. 6\%). Although nonsignificant, it is possible that these baseline differences between the two groups could have confounded the results, favoring CF use in healthier patients or those with less aggressive disease. However, even after adjusting for age and radiotherapy dose received, the negative association between dCRT with $\mathrm{CP}$ and survival remained. We also undertook sensitivity analyses using IPTW modeling on the overall cohort in an attempt to adjust for additional known confounders, which yielded similar results. We did not include the year of diagnosis in this model, given that $\mathrm{CP}$ had not been administered prior to 2014. This violation of the positivity assumption is a significant limitation of using IPTW and other propensity score methods to adjust for confounders in this study. However, given the small sample size, to our knowledge there were no alternative statistical methods which could be used. Randomized trials and/or pooled data from collaborative regis- tries would be required to confirm these findings. At this time, our study yields important precautionary data for contemporary practice.

\section{Conclusions}

OS outcomes for patients with locoregional esophageal or GEJ cancer are suboptimal. Notwithstanding the inherent limitations of a small retrospective study, our results suggest that concurrent dCRT with $\mathrm{CP}$ may be an inferior regimen compared to $\mathrm{CF}$ for patients with esophageal and GEJ cancers treated with a nonsurgical approach. Accordingly, we should be cautious about adopting $\mathrm{CP}$ as a standard regimen in the dCRT setting. nCRT $\mathrm{CP}$ and $\mathrm{CF}$ regimens yielded similar survival outcomes, and given the favorable toxicity profile associated with $\mathrm{CP}$, it remains the nCRT regimen of choice in the setting of TMT.

\section{Statement of Ethics}

This study was approved by the University Health Network institutional research ethics board (14-8075). The research ethics board waived the requirement to obtain individual patients' written consent, as this research posed a minimal risk to the subjects. Furthermore, many of the patients involved in the study will have deceased. This research complies with the guidelines for human studies and was conducted ethically in accordance with the World Medical Association Declaration of Helsinki.

\section{Conflict of Interest Statement}

C.H.L. has received a honorarium from Ipsen. R.W.J. has a consulting or advisory role for Ipsen and Novartis, and received research funding from AstraZeneca, Merck, Novartis, Eli Lilly, Boston Biomedical, and BMS. The other authors declare no conflict of interest.

\section{Funding Sources}

This research did not receive any specific grant from funding agencies in the public, commercial, or not-for-profit sectors.

\section{Author Contributions}

Di Maria Jiang: data curation, methodology, writing - original draft, review and editing. Hao-Wen Sim: conceptualization, data curation, methodology, statistical analysis, writing - review and editing. Osvaldo Espin-Garcia: statistical analysis, writing - review 
and editing. Bryan A. Chan: data curation, writing - review and editing. Akina Natori: data curation, writing - review and editing. Charles H. Lim: data curation, writing - review and editing. Stephanie Moignard: data curation, writing - review and editing. Eric X. Chen: data acquisition, writing - review and editing. Geoffrey Liu: data acquisition, writing - review and editing. Gail Darling: data acquisition, writing - review and editing. Carol J. Swallow: data acquisition, writing - review and editing. Savtaj Brar: data acquisition, writing - review and editing. James Brierley: data acquisition, writ- ing - review and editing. Jolie Ringash: data acquisition, writing review and editing. Rebecca Wong: data acquisition, writing - review and editing. John Kim: data acquisition, writing - review and editing. Patrik Rogalla: data acquisition, writing - review and editing. Sara Hafezi-Bakhtiari: data acquisition, writing - review and editing. Jennifer J. Knox: data acquisition, writing - review and editing. Raymond W. Jang: conceptualization, data acquisition, writing - review and editing. Elena Elimova: conceptualization, data acquisition, methodology writing - review and editing.

\section{Appendix 1}

Reasons for Not Undergoing Surgical

Resection in Patients Treated with Neoadjuvant and Definitive Chemoradiation Concurrent with CF and CP

\section{References}

1 van Hagen P, Hulshof MC, van Lanschot JJ, Steyerberg EW, van Berge Henegouwen MI, Wijnhoven BPL, et al. Preoperative chemoradiotherapy for esophageal or junctional cancer. N Engl J Med. 2012;366(22):2074-84.

2 Dubecz A, Gall I, Solymosi N, Schweigert M, Peters JH, Feith M, et al. Temporal trends in long-term survival and cure rates in esophageal cancer: a SEER database analysis. J Thorac Oncol. 2012;7(2):443-7.

3 Walsh TN, Noonan N, Hollywood D, Kelly A, Keeling N, Hennessy TP. A comparison of multimodal therapy and surgery for esophageal adenocarcinoma. N Engl J Med. 1996; 335(7):462-7.

4 Tepper J, Krasna MJ, Niedzwiecki D, Hollis D, Reed CE, Goldberg R, et al. Phase III trial of trimodality therapy with cisplatin, fluorouracil, radiotherapy, and surgery compared with surgery alone for esophageal cancer: CALGB 9781. J Clin Oncol. 2008;26(7):108692.

5 Shapiro J, van Lanschot, JJB, Hulshof MCCM, van Hagen $\mathrm{P}$, van Berge Henegouwen MI, Wijnhoven BPL, et al. Neoadjuvant chemoradiotherapy plus surgery versus surgery alone for oesophageal or junctional cancer (CROSS): long-term results of a randomised controlled trial. Lancet Oncol. 2015;16(9):1090-8.

6 Cooper JS, Guo MD, Herskovic A, Macdonald JS, Martenson JA Jr, Al-Sarraf M, et al. Chemoradiotherapy of locally advanced esophageal cancer: long-term follow-up of a prospective randomized trial (RTOG 85-01).

\begin{tabular}{lll}
\hline Neoadjuvant chemoradiation $(n=10)$ & $\mathrm{CF}(n=5)$ & $\mathrm{CP}(n=5)$ \\
Toxicity during chemoradiation & 2 & 1 \\
Progressive disease & 3 & 4 \\
Definitive chemoradiation $(n=26)$ & $\mathrm{CF}(n=18)$ & $\mathrm{CP}(n=8)$ \\
Proximal tumor location & 10 & 3 \\
Age 80 years/comorbidities/poor performance status & 2 & 5 \\
Extent of nodal disease & 6 & 0 \\
\hline
\end{tabular}

Radiation Therapy Oncology Group. JAMA. 1999;281(17):1623-7.

7 Minsky BD, Pajak TF, Ginsberg RJ, Pisansky TM, Martenson J, Komaki R, et al. INT 0123 (Radiation Therapy Oncology Group 94-05) phase III trial of combined-modality therapy for esophageal cancer: high-dose versus standard-dose radiation therapy. J Clin Oncol. 2002;20(5):1167-74.

8 National Comprehensive Cancer Network. Esophageal and esophagogastric junction cancers (version 1.2019) [Internet] [accessed 2019 May 13]. 2019.

9 Peduzzi P, Concato J, Kemper E, Holford TR, Feinstein AR. A simulation study of the number of events per variable in logistic regression analysis. J Clin Epidemiol. 1996;49(12):1373-9.

10 Blom RL, Sosef MN, Nap M, Lammering G, van den Berkmortel F, Hulshof MC, et al. Comparison of two neoadjuvant chemoradiotherapy regimens in patients with potentially curable esophageal carcinoma. Dis Esophagus. 2014;27(4):380-7.

11 Buckstein M, Rhome R, Ru M, Moshier E. Neoadjuvant chemoradiation radiation dose levels for surgically resectable esophageal cancer: predictors of use and outcomes. Dis Esophagus. 2018;31(5):dox148.

12 Qu XM, Biagi JJ, Hopman WM, Mahmud A. Shifting practice in definitive chemoradiation for localized esophageal cancer. Curr Oncol. 2017;24(5):e379-87.

13 Honing J, Smit JK, Muijs CT, Burgerhof JG, de Groot JW, Paardekooper G, et al. A com- parison of carboplatin and paclitaxel with cisplatinum and 5-fluorouracil in definitive chemoradiation in esophageal cancer patients. Ann Oncol. 2014;25(3):638-43.

14 Münch S, Pigorsch SU, Devečka M, Dapper $\mathrm{H}$, Weichert W, Friess H, et al. Comparison of definite chemoradiation therapy with carboplatin/paclitaxel or cisplatin/5-fluoruracil in patients with squamous cell carcinoma of the esophagus. Radiat Oncol. 2018;13(1):139.

15 Shao MS, Wong AT, Schwartz D, Weiner JP, Schreiber D. Definitive or preoperative chemoradiation therapy for esophageal cancer: patterns of care and survival outcomes. Ann Thorac Surg. 2016;101(6):2148-54.

16 Brower JV, Chen S, Bassetti MF, Yu M, Harari PM, Ritter MA, et al. Radiation dose escalation in esophageal cancer revisited: a contemporary analysis of the National Cancer Data Base, 2004 to 2012. Int J Radiat Oncol Biol Phys. 2016;96(5):985-93.

17 Liang J, Bi N, Wu S, Chen M, Lv C, Zhao L, et al. Etoposide and cisplatin versus paclitaxel and carboplatin with concurrent thoracic radiotherapy in unresectable stage III nonsmall cell lung cancer: a multicenter randomized phase III trial. Ann Oncol. 2017;28(4): $777-83$

18 Guan J, Li Q, Zhang Y, Xiao N, Chen M, Zhang $\mathrm{Y}$, et al. A meta-analysis comparing cisplatin-based to carboplatin-based chemotherapy in moderate to advanced squamous cell carcinoma of head and neck (SCCHN). Oncotarget. 2016;7(6):7110-9. 